\title{
Kinetic Resolution of Racemic Secondary Alcohols Catalyzed by Chiral Diaminodiphosphine-Ir(I) complexes
}

\author{
Yan-Yun Li, Xue-Qin Zhang, Zhen-Rong Dong, Wei-Yi Shen, Gui Chen, Jing-Xing Gao* \\ State Key Laboratory of Physical Chemistry of Solid Surfaces and Department of Chemistry, College of Chemistry and \\ Chemical Engineering, Xiamen University, Xiamen 361005, Fujian, P. R. China \\ cuihua@jingxian.xmu.edu.cn, jxgao@xmu.edu.cn
}

\section{List of the Contents of the Supporting Information}

1. General methods

Page S1

2. A typical procedure for oxidative kinetic resolution of racemic secondary alcohol

Page S1

3. GC analytical data for chiral aromatic alcohols

Page S2

\section{General methods}

All experiments were carried out in a nitrogen atmosphere with Schlenk techniques, all solvents were dried and purified according to standard methods before use. Racemic 1-phenylethanol (Aldrich) was used without further purification and all other racemic alcohols were synthesized by $\mathrm{NaBH}_{4}$ reduction in ethanol from their respective ketones. Enantiomeric excess in each product was determined by GC using a chiral column (Chiraldex G-TA column).

\section{A typical procedure for oxidative kinetic resolution of racemic secondary alcohols}

The oxidative kinetic resolution was conducted in a schlenk tube. An example is given by oxidative kinetic resolution of racemic 1-phenylethanol using chiral metal complex in situ generated from $(R, R)-1$ and $[\mathrm{Ir}(\mathrm{COD}) \mathrm{Cl}]_{2}$ as catalyst precursor.

To a mixture of $(R, R)-1(0.005 \mathrm{mmol})$ and $[\operatorname{Ir}(\mathrm{COD}) \mathrm{Cl}]_{2}(0.0025 \mathrm{mmol})$ was added anhydrous acetone $(10 \mathrm{~mL})$ under nitrogen. After the solution was stirred for $1 \mathrm{~h}$ at $25^{\circ} \mathrm{C}$, a solution of $\mathrm{KOH}$ in ${ }^{i} \mathrm{PrOH}$, and then racemic 1-phenylethanol $(1 \mathrm{mmol})$ were slowly added. The solution was stirred at $25{ }^{\circ} \mathrm{C}$ for $8 \mathrm{~h}$. Samples were taken out of the reaction solution, passed through a column of silica. The chemical yield and ee of products were determined by GC using a chiral column (Chiraldex G-TA column). 


\section{GC analytical data for chiral aromatic alcohols}<smiles>CC(O)c1ccccc1</smiles>

$(\mathbf{2 a})$

GC analysis: Chiraldex G-TA column, $0.25 \mathrm{~mm} \times 30 \mathrm{~m}$, column temperature $=90{ }^{\circ} \mathrm{C}$ (isothermal), inject temperature $=250{ }^{\circ} \mathrm{C}$, detector temperature $=250{ }^{\circ} \mathrm{C}$, inlet pressure $=0.1 \mathrm{MPa} ; \mathrm{t}_{3}=13.33 \mathrm{~min}, \mathrm{t}_{1}=15.73$ min, $t_{2}=16.00$ min. $\left(t_{3}:\right.$ retention time of ketone; $t_{1}, t_{2}:$ retention time of chiral alcohol. $)$

Racemic 2a:

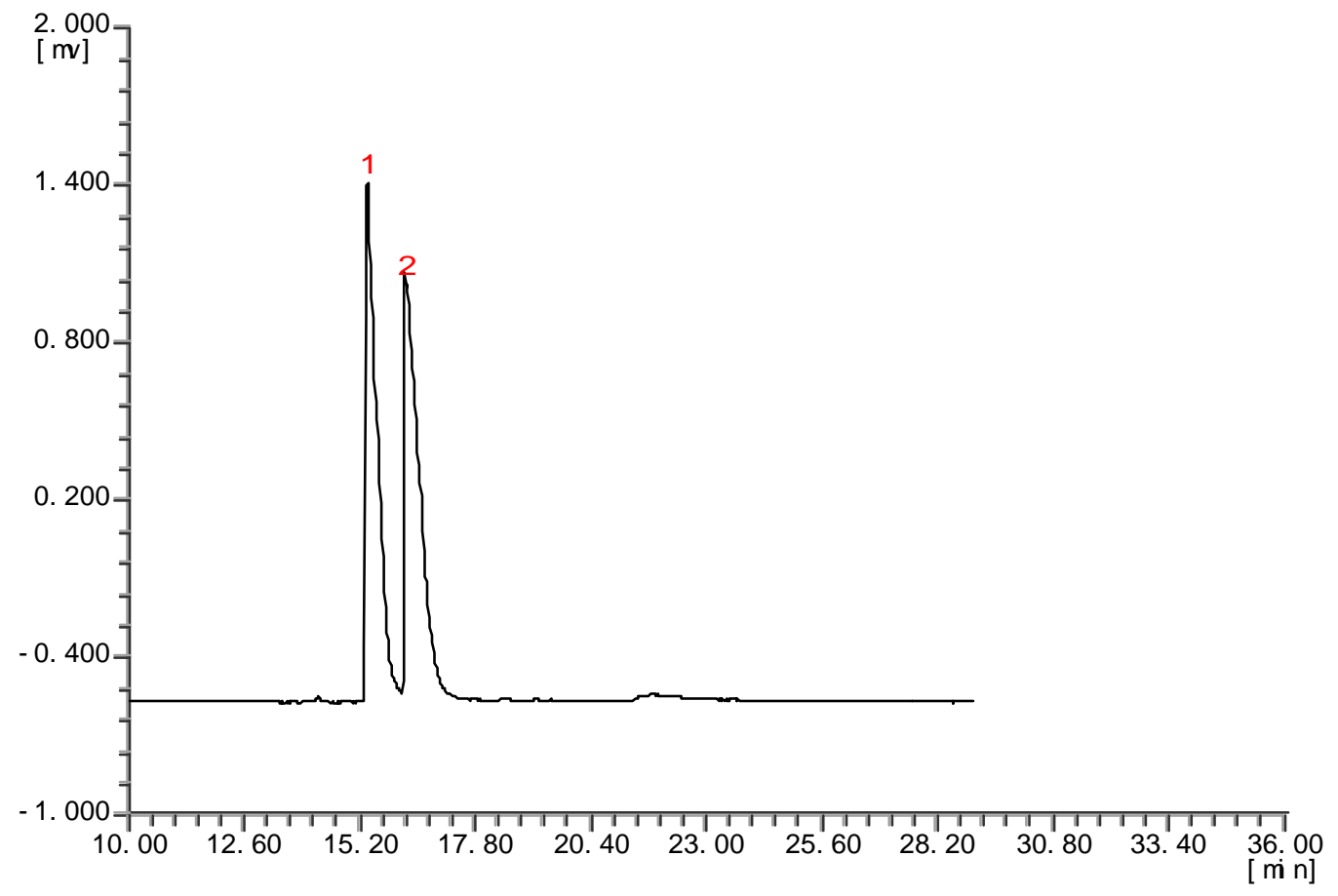

Resolved 2a (Table 2, run 1):

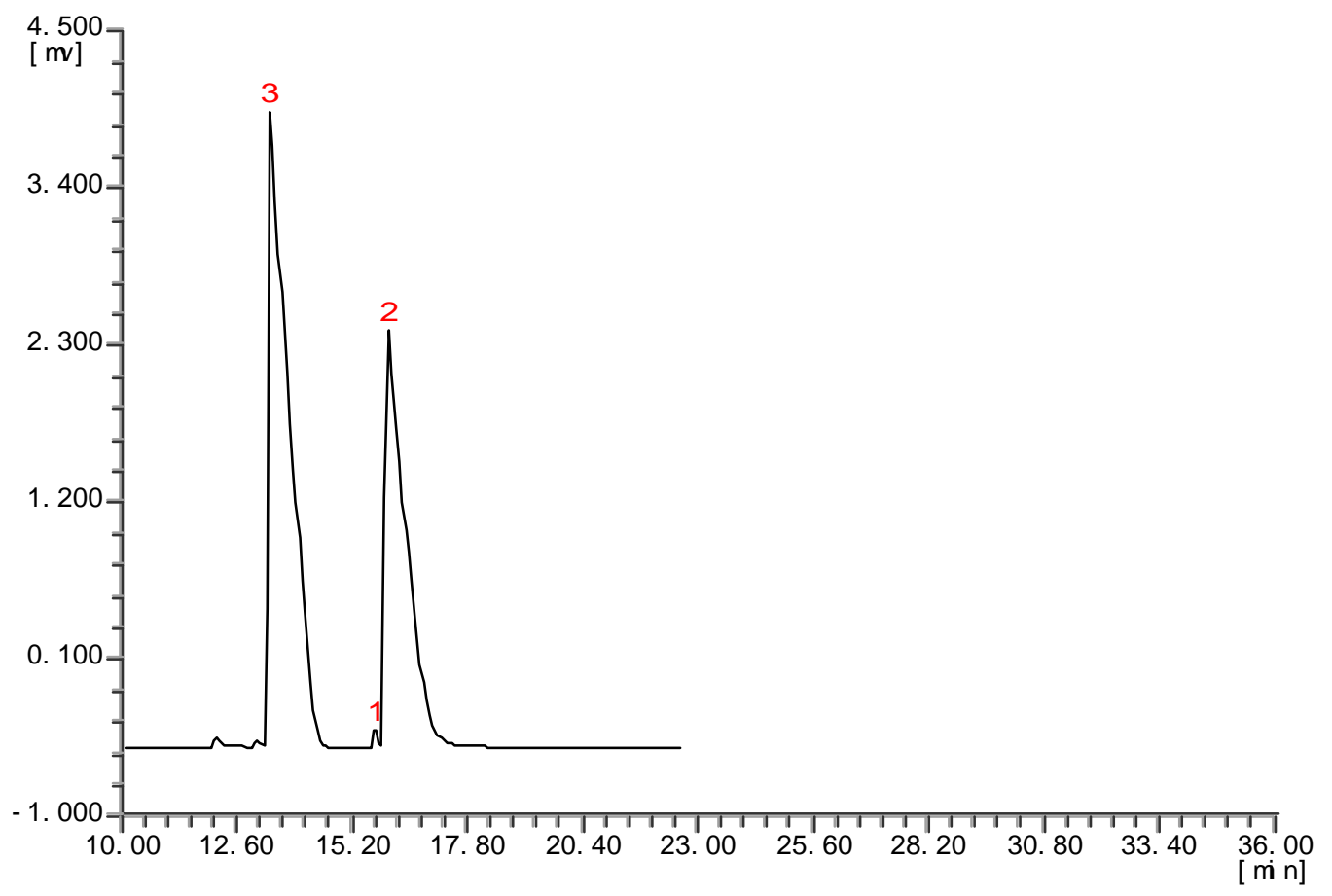


<smiles>CCC(O)c1ccccc1</smiles>

(2b)

GC analysis: Chiraldex G-TA column, $0.25 \mathrm{~mm} \times 30 \mathrm{~m}$, column temperature $=100{ }^{\circ} \mathrm{C}$ (isothermal), inject temperature $=250{ }^{\circ} \mathrm{C}$, detector temperature $=250{ }^{\circ} \mathrm{C}$, inlet pressure $=0.1 \mathrm{MPa} ; \mathrm{t}_{3}=11.07 \mathrm{~min}, \mathrm{t}_{1}=$ $13.18 \mathrm{~min}, \mathrm{t}_{2}=13.78 \mathrm{~min}$. ( $\mathrm{t}_{3}$ : retention time of ketone; $\mathrm{t}_{1}, \mathrm{t}_{2}$ : retention time of chiral alcohol.)

Racemic 2b:

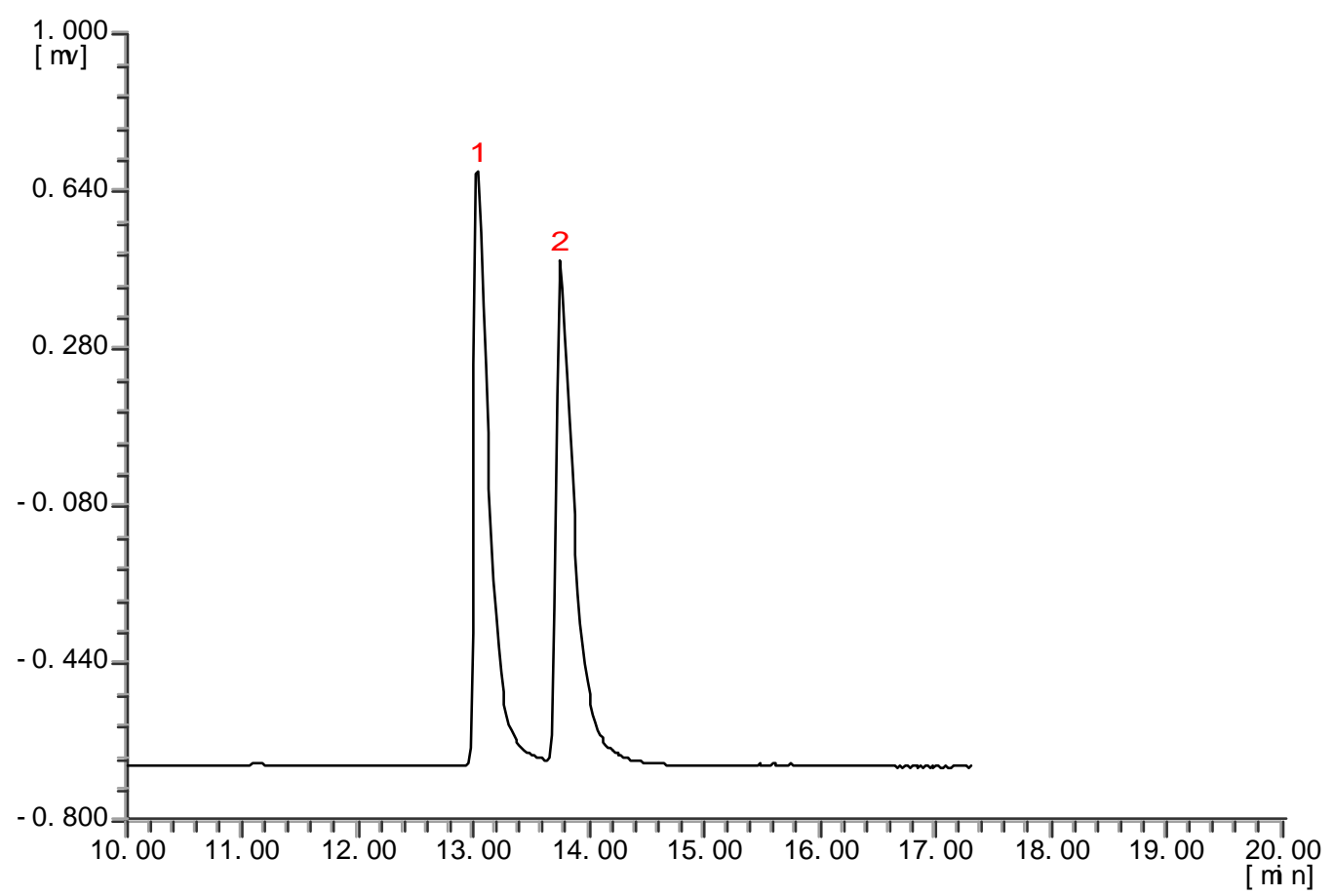

Resolved 2b (Table 2, run 2):

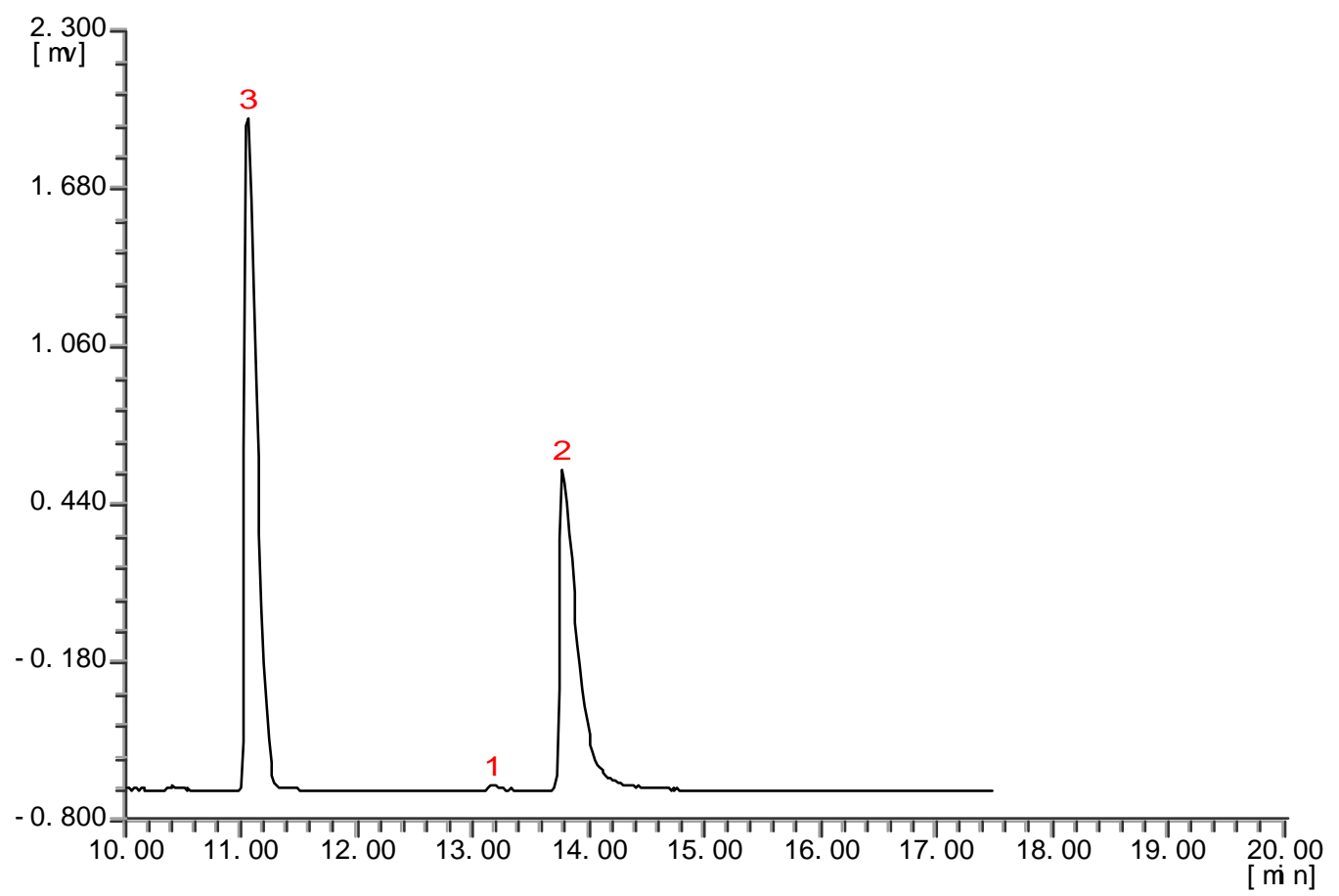


<smiles>CCCC(O)c1ccccc1</smiles>

(2c)

GC analysis: Chiraldex G-TA column, $0.25 \mathrm{~mm} \times 30 \mathrm{~m}$, column temperature $=110{ }^{\circ} \mathrm{C}$ (isothermal), inject temperature $=250{ }^{\circ} \mathrm{C}$, detector temperature $=250{ }^{\circ} \mathrm{C}$, inlet pressure $=0.1 \mathrm{MPa} ; \mathrm{t}_{3}=10.02 \mathrm{~min}, \mathrm{t}_{1}=$ 12.56 min, $t_{2}=13.03$ min. ( $t_{3}:$ retention time of ketone; $t_{1}, t_{2}:$ retention time of chiral alcohol.)

Racemic 2c:

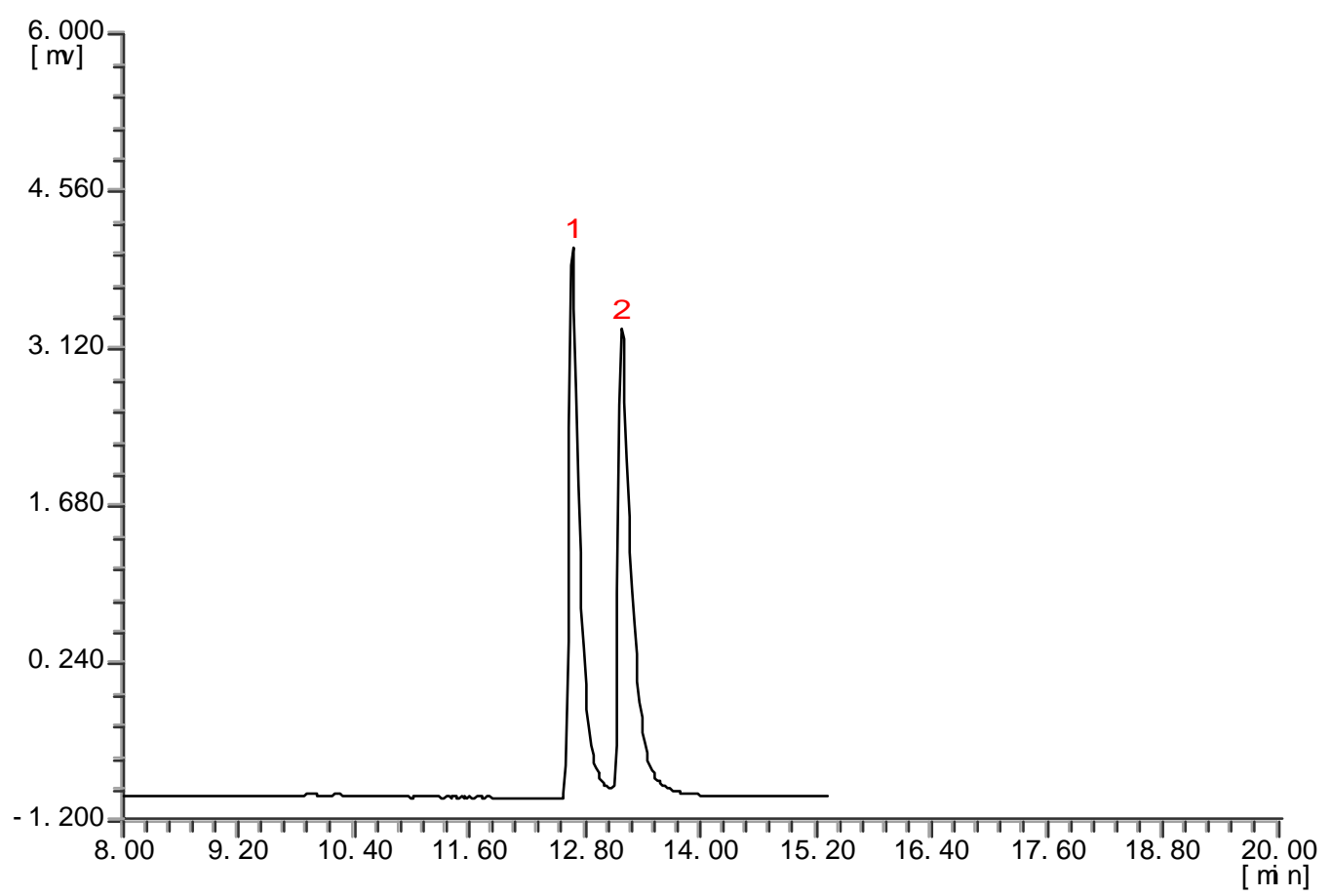

Resolved 2c (Table 2, run 3):

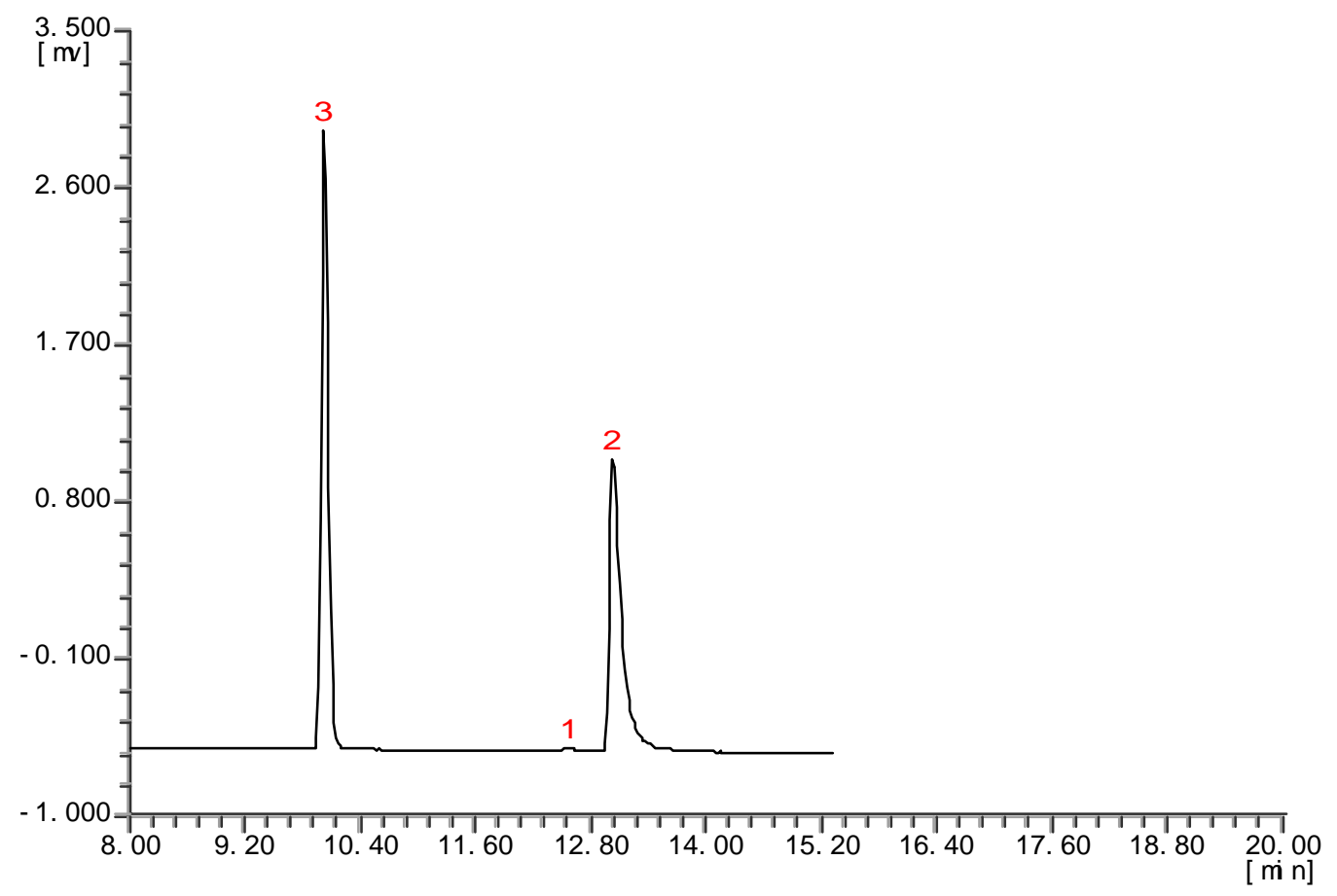


<smiles>CCCCC(O)c1ccccc1</smiles>

(2d)

GC analysis: Chiraldex G-TA column, $0.25 \mathrm{~mm} \times 30 \mathrm{~m}$, column temperature $=110{ }^{\circ} \mathrm{C}$ (isothermal), inject temperature $=250{ }^{\circ} \mathrm{C}$, detector temperature $=250{ }^{\circ} \mathrm{C}$, inlet pressure $=0.1 \mathrm{MPa} ; \mathrm{t}_{3}=16.60 \mathrm{~min}, \mathrm{t}_{1}=$ $20.98 \mathrm{~min}, \mathrm{t}_{2}=21.93 \mathrm{~min}$. ( $\mathrm{t}_{3}:$ retention time of ketone; $\mathrm{t}_{1}, \mathrm{t}_{2}$ : retention time of chiral alcohol.)

Racemic 2d:

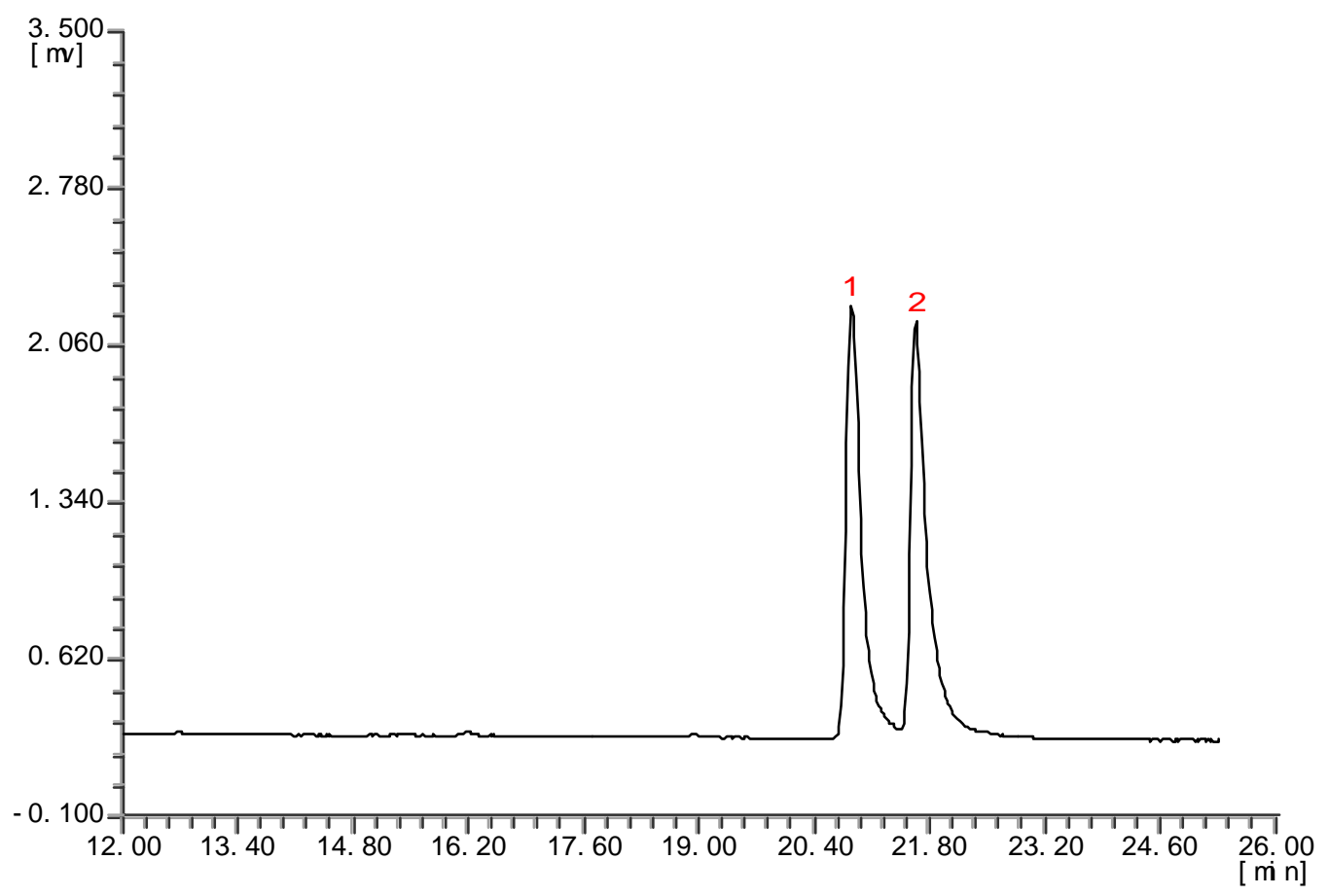

Resolved 2d (Table 2, run 4):

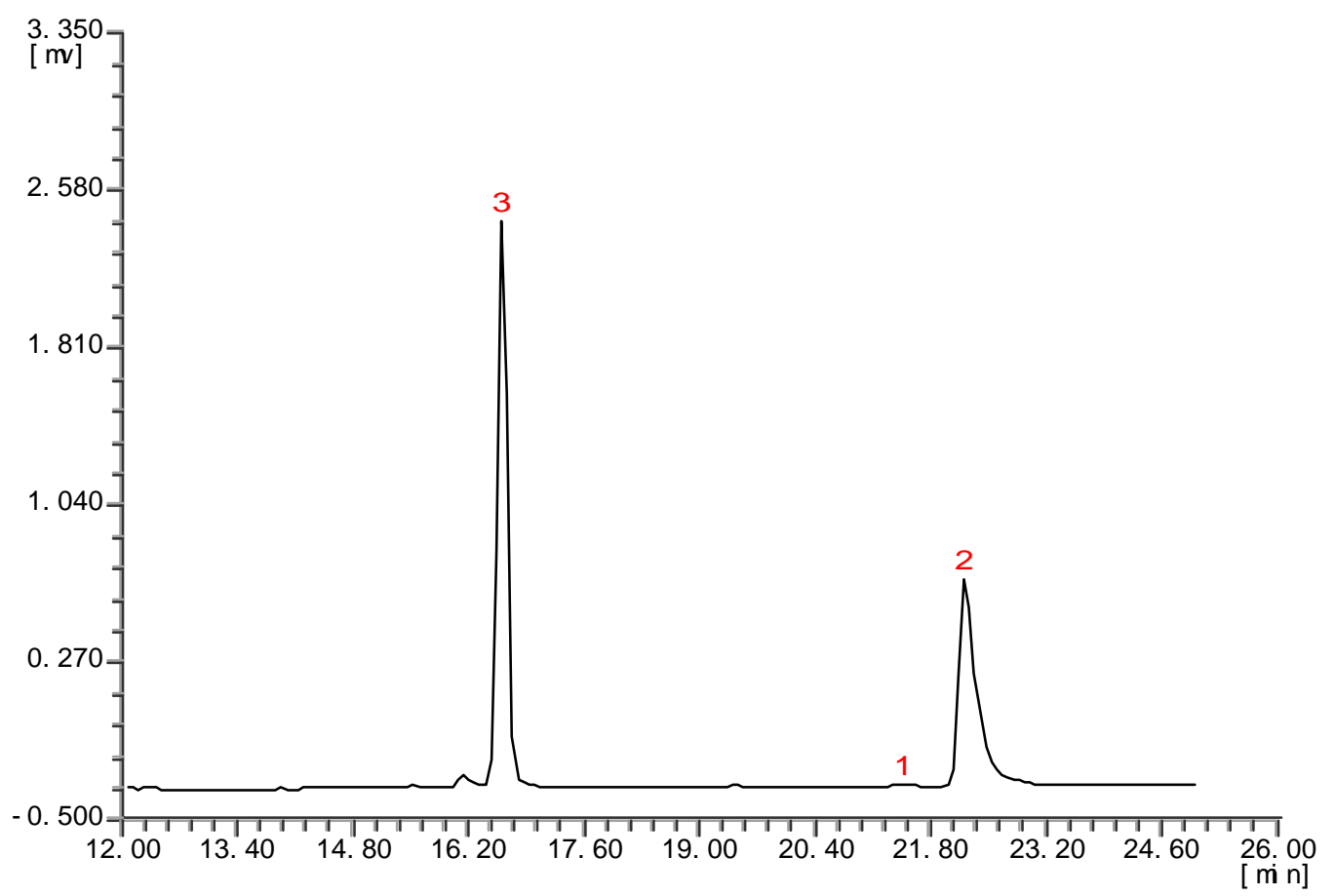


<smiles>CC(C)C(O)c1ccccc1</smiles>

$(\mathbf{2 e})$

GC analysis: Chiraldex G-TA column, $0.25 \mathrm{~mm} \times 30 \mathrm{~m}$, column temperature $=95^{\circ} \mathrm{C}$ (isothermal), inject temperature $=250{ }^{\circ} \mathrm{C}$, detector temperature $=250{ }^{\circ} \mathrm{C}$, inlet pressure $=0.1 \mathrm{MPa} ; \mathrm{t}_{3}=13.48 \mathrm{~min}, \mathrm{t}_{1}=18.91$ min, $t_{2}=19.73$ min. $\left(t_{3}\right.$ : retention time of ketone; $t_{1}, t_{2}$ : retention time of chiral alcohol.)

Racemic 2e:

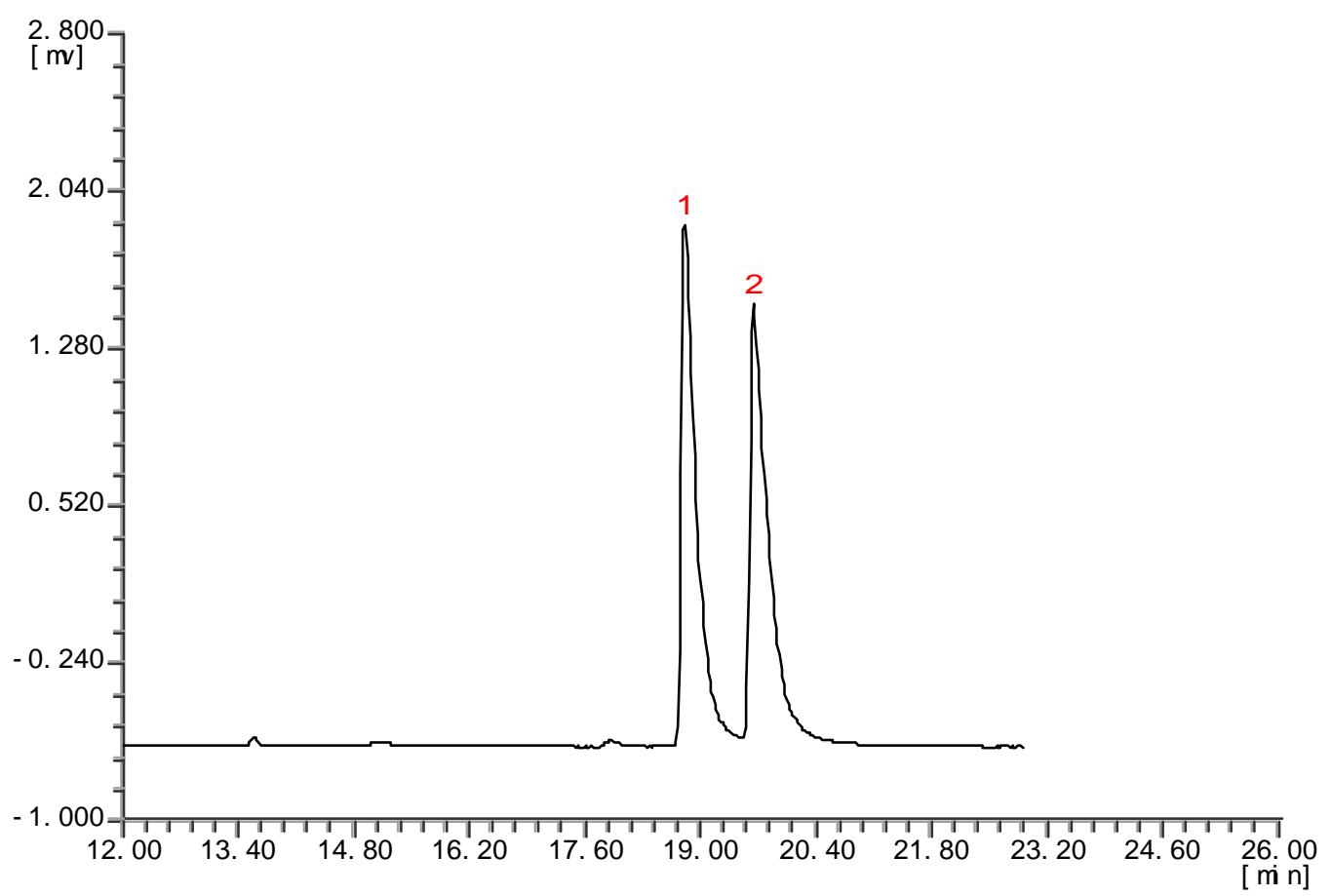

Resolved 2e (Table 2, run 5):

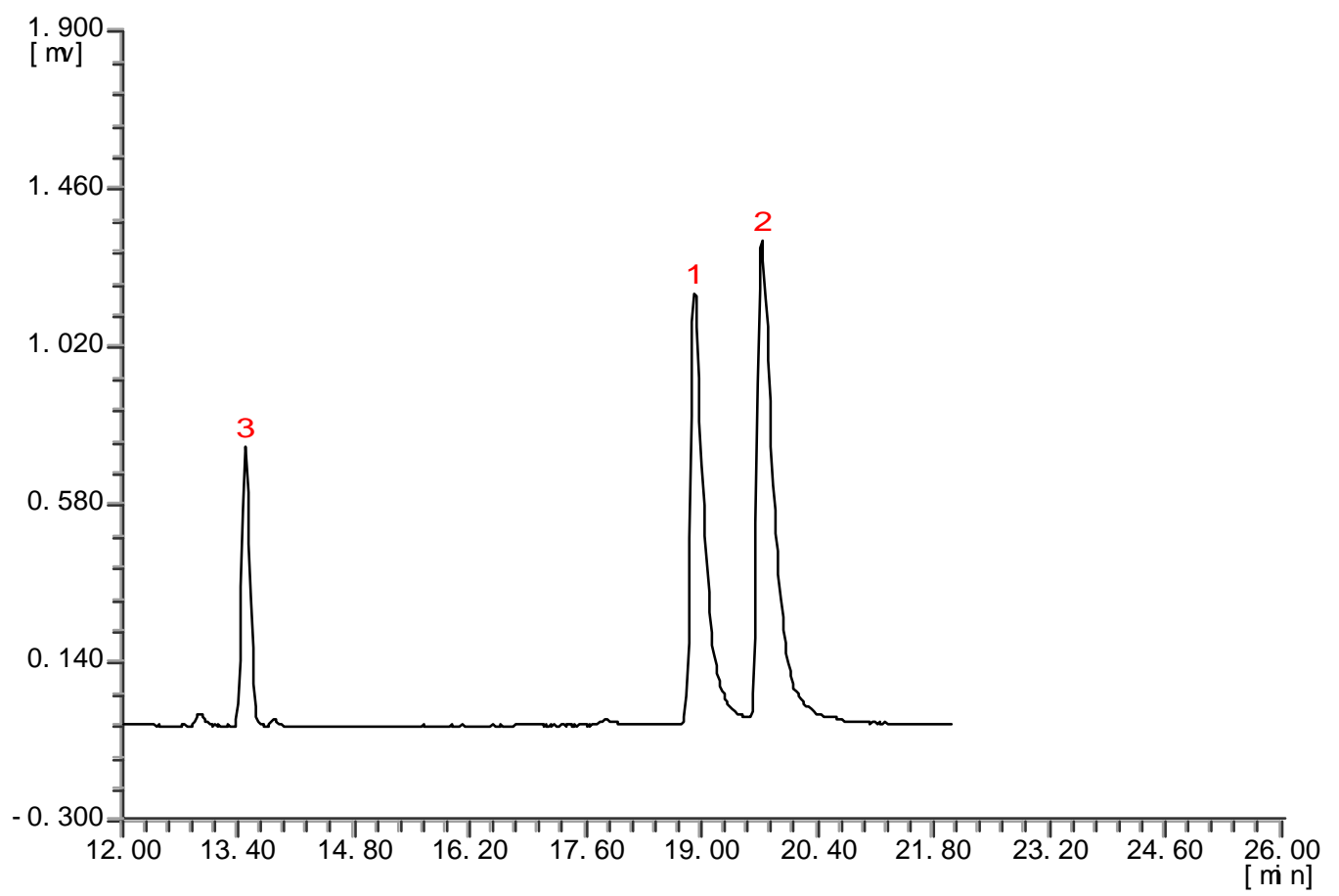


<smiles>Cc1ccccc1C(C)O</smiles>

GC analysis: Chiraldex G-TA column, $0.25 \mathrm{~mm} \times 30 \mathrm{~m}$, column temperature $=110{ }^{\circ} \mathrm{C}$ (isothermal), inject temperature $=250{ }^{\circ} \mathrm{C}$, detector temperature $=250{ }^{\circ} \mathrm{C}$, inlet pressure $=0.1 \mathrm{MPa} ; \mathrm{t}_{3}=7.60 \mathrm{~min}, \mathrm{t}_{1}=$ $11.22 \mathrm{~min}, \mathrm{t}_{2}=12.58 \mathrm{~min}$. ( $\mathrm{t}_{3}$ : retention time of ketone; $\mathrm{t}_{1}, \mathrm{t}_{2}$ : retention time of chiral alcohol.)

Racemic $\boldsymbol{o - 2 f}$ :

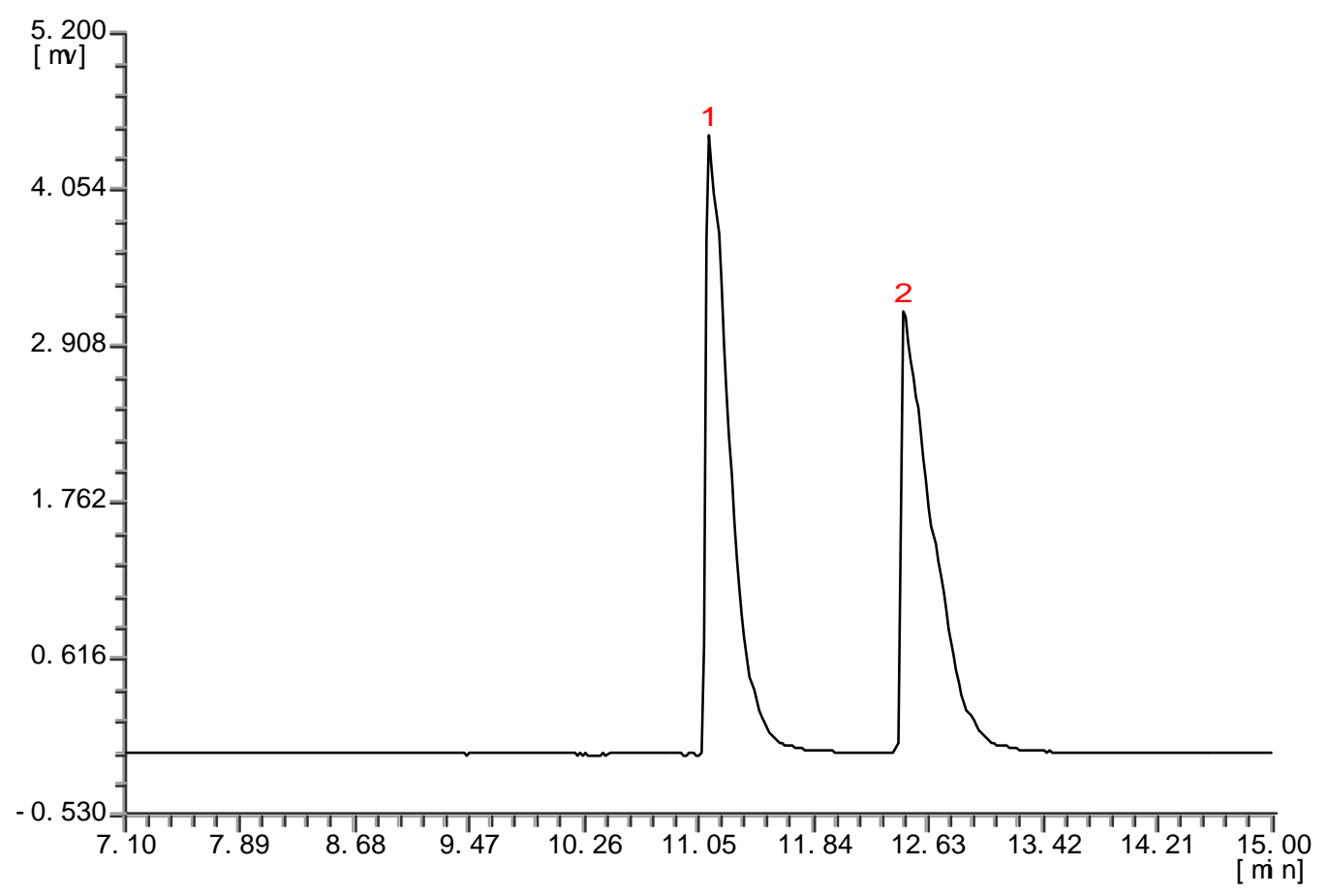

Resolved $\boldsymbol{o}$-2f (Table 2, run 6):

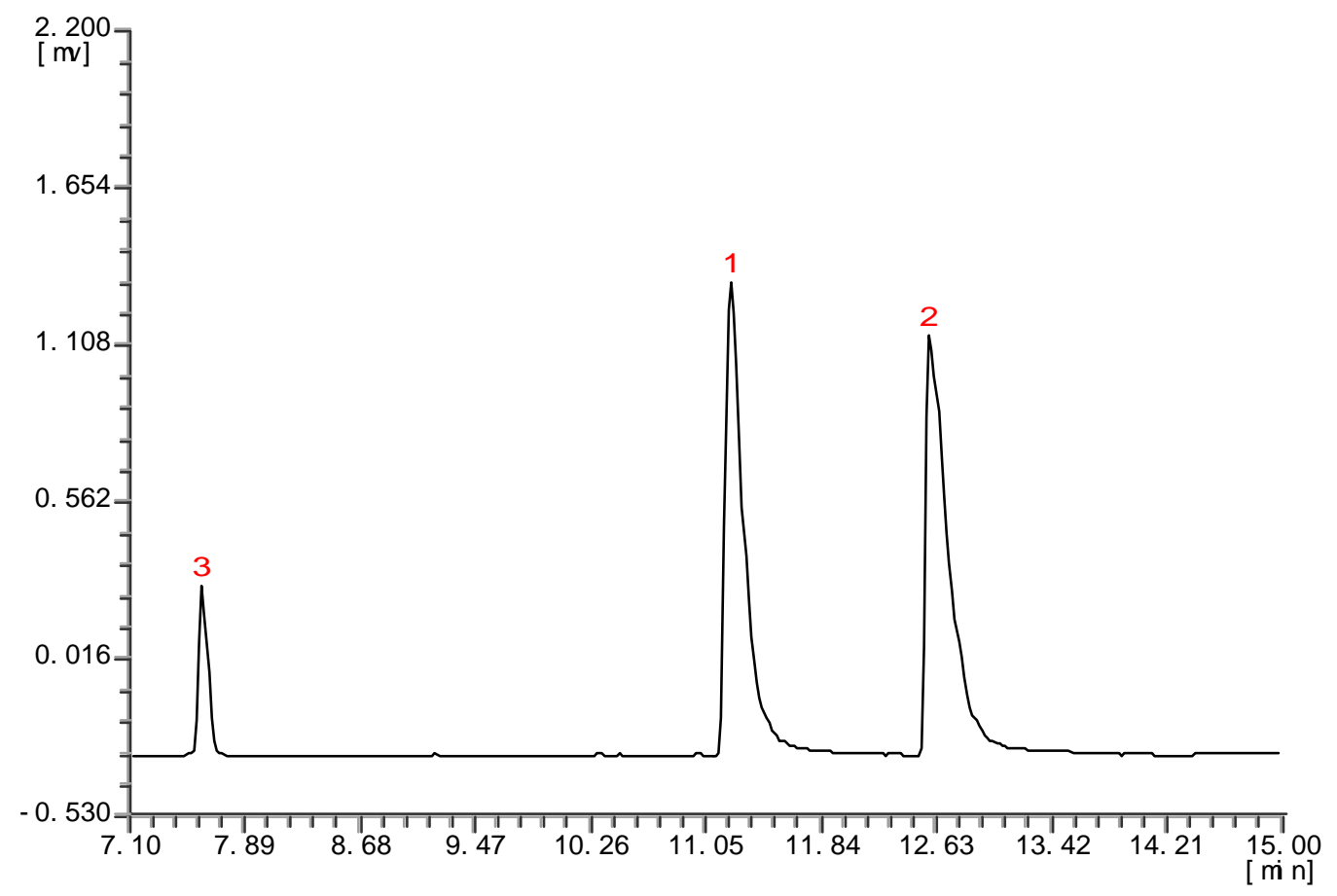


<smiles>Cc1cccc(C(C)O)c1</smiles>

GC analysis: Chiraldex G-TA column, $0.25 \mathrm{~mm} \times 30 \mathrm{~m}$, column temperature $=110{ }^{\circ} \mathrm{C}$ (isothermal), inject temperature $=250{ }^{\circ} \mathrm{C}$, detector temperature $=250{ }^{\circ} \mathrm{C}$, inlet pressure $=0.1 \mathrm{MPa} ; \mathrm{t}_{3}=9.14 \mathrm{~min}, \mathrm{t}_{1}=$ $9.94 \mathrm{~min}, \mathrm{t}_{2}=10.54 \mathrm{~min}$. ( $\mathrm{t}_{3}$ : retention time of ketone; $\mathrm{t}_{1}, \mathrm{t}_{2}$ : retention time of chiral alcohol.)

Racemic $m$-2f:

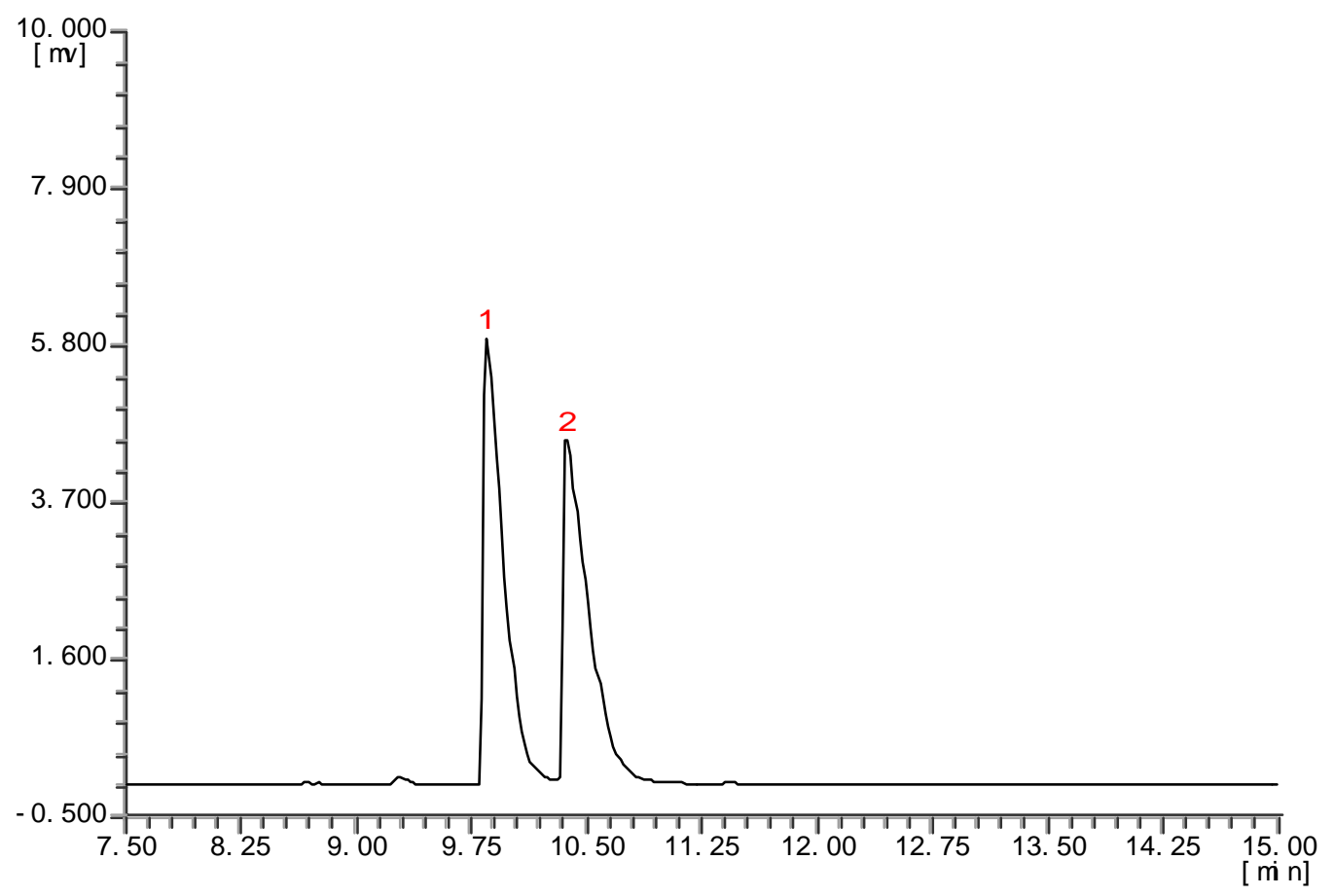

Resolved $m$-2f (Table 2, run 7):

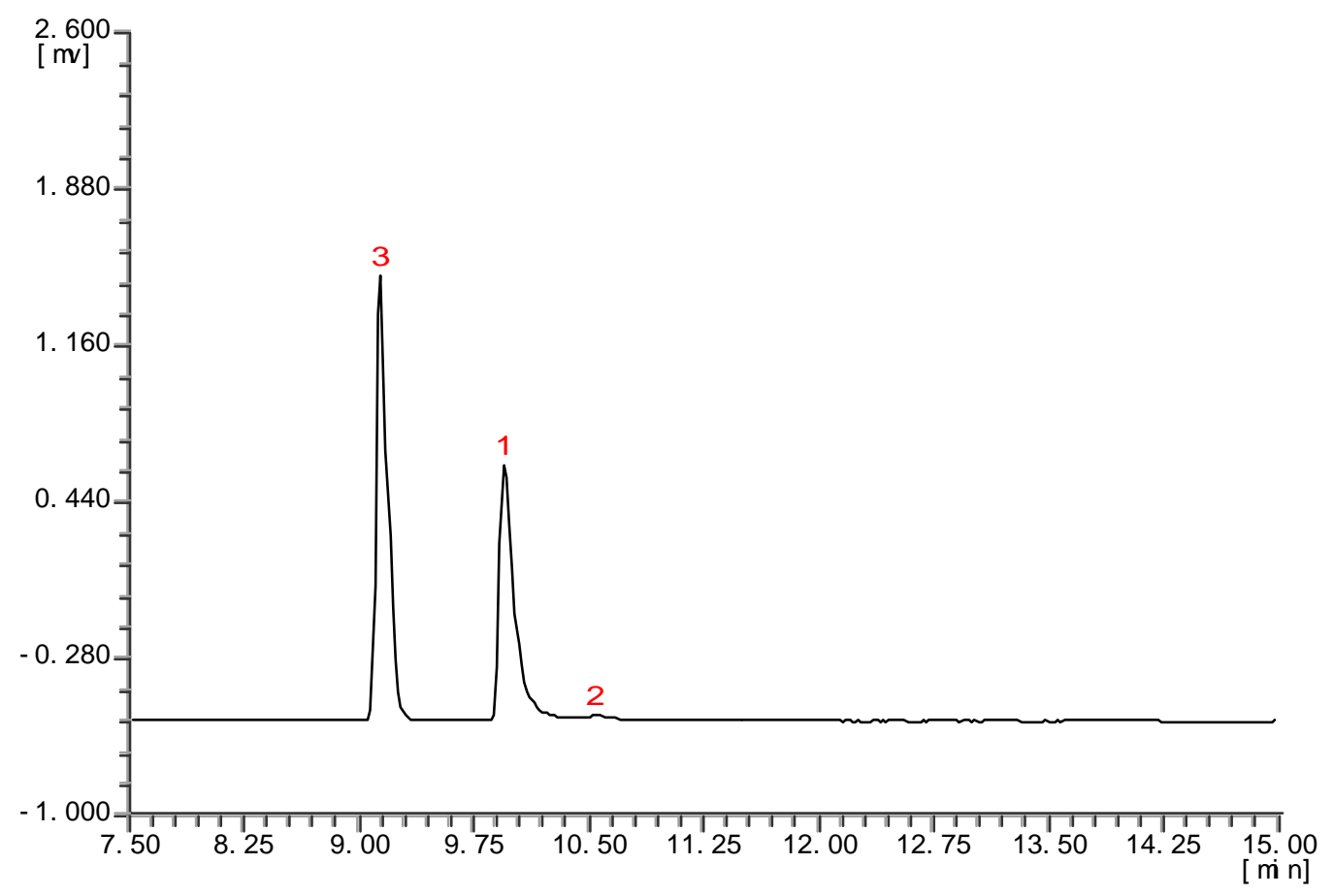


<smiles>CC(O)c1cccc(Cl)c1</smiles>

GC analysis: Chiraldex G-TA column, $0.25 \mathrm{~mm} \times 30 \mathrm{~m}$, column temperature $=110{ }^{\circ} \mathrm{C}$ (isothermal), inject temperature $=250{ }^{\circ} \mathrm{C}$, detector temperature $=250{ }^{\circ} \mathrm{C}$, inlet pressure $=0.1 \mathrm{MPa} ; \mathrm{t}_{3}=12.72 \mathrm{~min}, \mathrm{t}_{1}=$ $24.00 \mathrm{~min}, \mathrm{t}_{2}=28.10 \mathrm{~min}$. ( $\mathrm{t}_{3}:$ retention time of ketone; $\mathrm{t}_{1}, \mathrm{t}_{2}$ : retention time of chiral alcohol.)

Racemic $m$-2g:

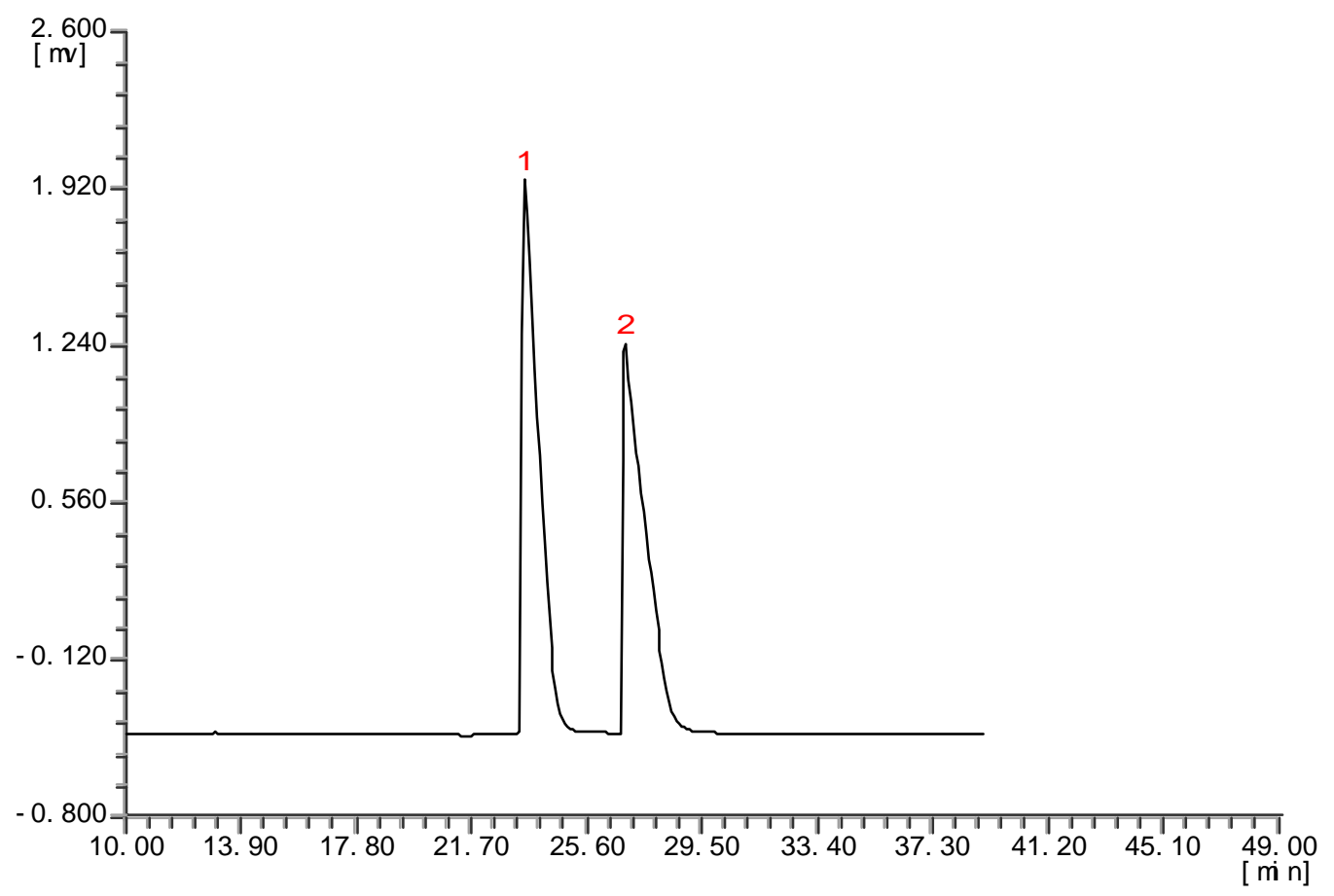

Resolved $m$-2g (Table 2, run 8):

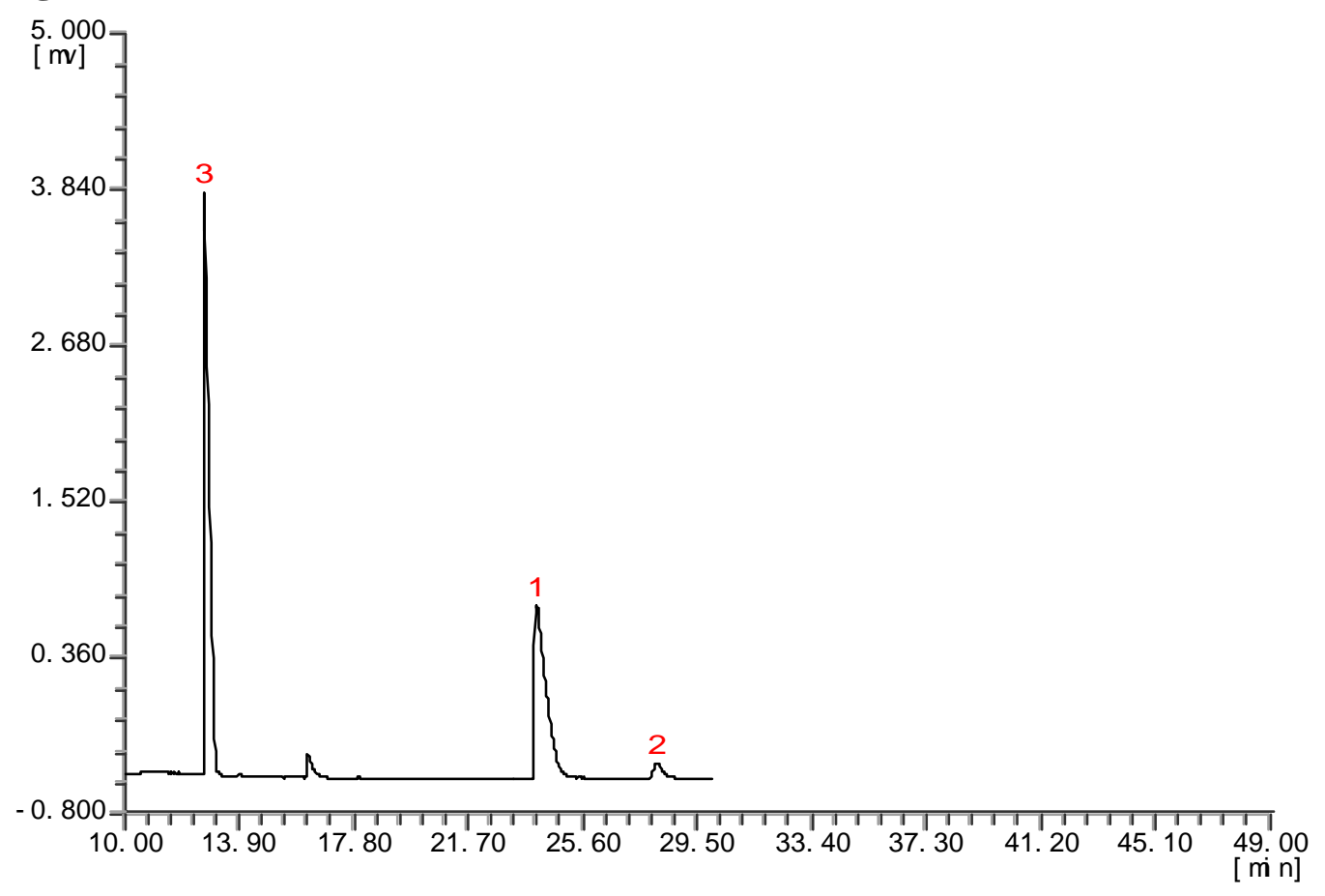


<smiles>CC(O)c1ccc(Cl)cc1</smiles>

GC analysis: Chiraldex G-TA column, $0.25 \mathrm{~mm} \times 30 \mathrm{~m}$, column temperature $=110{ }^{\circ} \mathrm{C}$ (isothermal), inject temperature $=250{ }^{\circ} \mathrm{C}$, detector temperature $=250{ }^{\circ} \mathrm{C}$, inlet pressure $=0.1 \mathrm{MPa} ; \mathrm{t}_{3}=10.87 \mathrm{~min}, \mathrm{t}_{1}=$ $18.31 \mathrm{~min}, \mathrm{t}_{2}=20.19 \mathrm{~min}$. ( $\mathrm{t}_{3}$ : retention time of ketone; $\mathrm{t}_{1}, \mathrm{t}_{2}$ : retention time of chiral alcohol.)

Racemic $p$-2g:

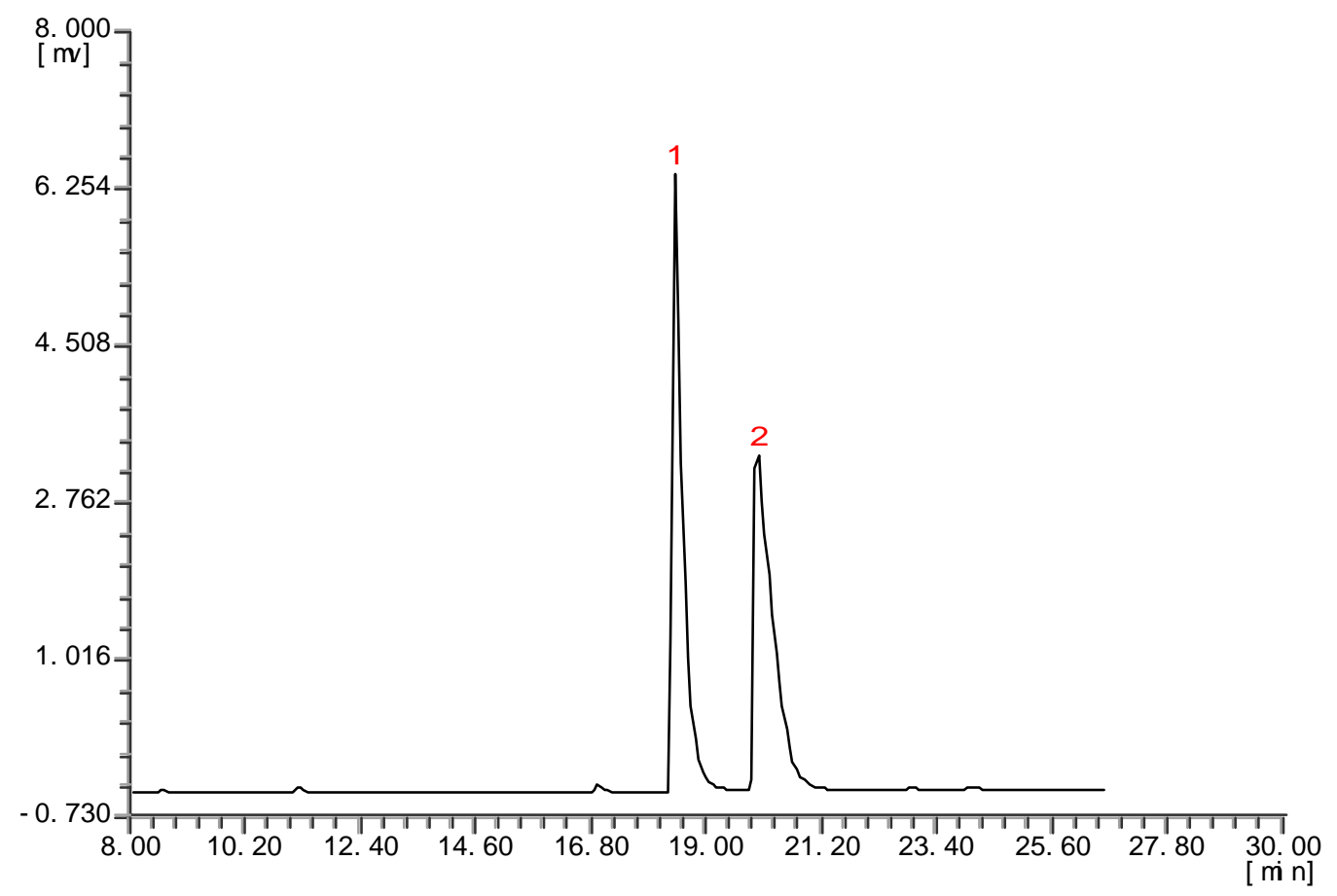

Resolved $p$-2g (Table 2, run 9):

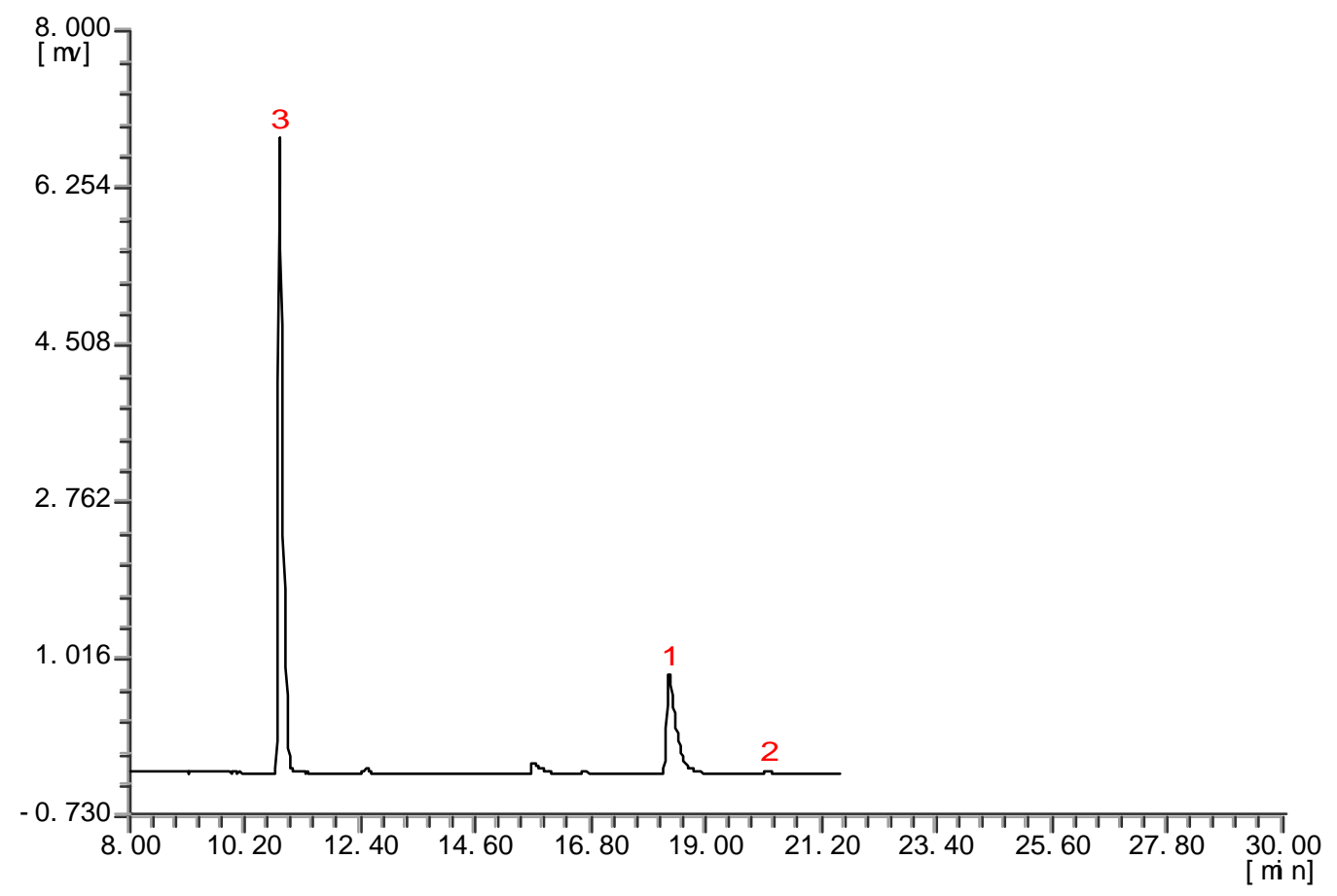

